\title{
High Angular Resolution Studies of Stellar Atmospheres
}

\author{
Chris A. Haniff \\ Astrophysics Group, Cavendish Laboratory, Madingley Road, \\ Cambridge, CB3 OHE, UK.
}

\begin{abstract}
The advent of long-baseline optical and infrared interferometers has meant that spatially resolved studies of stellar atmospheres have now become routinely possible. While prototype arrays, which have typically operated with short baselines and limited sensitivity, have produced exciting preliminary results, it is the development of larger dedicated facility arrays, such as the CHARA, Keck, and VLT interferometers, that offer the best prospects for advancing astrophysics. In this paper I review the possibilities and limitations of interferometric studies of stellar atmospheres, and highlight some recent results from optical/IR spatial interferometry.
\end{abstract}

\section{Introduction}

The small apparent size of all but the nearest and largest stars has meant that studies of the spatially resolved structure of stellar atmospheres have, until very recently, not featured prominently in the astronomical literature. A cursory glance at the typical scales expected for different types of stars within the local neighbourhood (see Table 1) shows that even with HST-type angular resolution (for $D=2.4 \mathrm{~m}, \lambda / D=43$ mas) only the nearest supergiant stars will exhibit resolvable disks. Although approaches such as spectroscopy and time resolved

Table 1. Typical angular sizes for different stellar types

\begin{tabular}{lcc}
\hline Stellar type & Typical radius $/ R_{\odot}$ & Angular diameter at $250 \mathrm{pc} / \mathrm{mas}$ \\
\hline Dwarfs & $0.2-15$ & $0.007-0.56$ \\
Giants & $3-100$ & $0.11-3.7$ \\
Supergiants & $20-700$ & $0.7-26$ \\
\hline \hline
\end{tabular}

photometry can explore spatial scales below the diffraction limit, it is clear that the most direct route to probing the spatial structure of distant stellar atmospheres will be through interferometric imaging with very long baseline $(B \geq 100 \mathrm{~m})$ optical and near-infrared interferometers. At these wavelengths suitable angular resolution becomes available - a $100 \mathrm{~m}$ interferometer operating at $0.5 \mu \mathrm{m}$ has a fringe spacing of 1 milliarcsecond - and one can realistically envisage probing the global, and local, structures of a reasonable sample of the larger stars in the Solar neighbourhood.

Interferometric measurements at other wavelengths have already been useful here (see, for example, the radio observations of Lim et al. (1998), and the mid- 


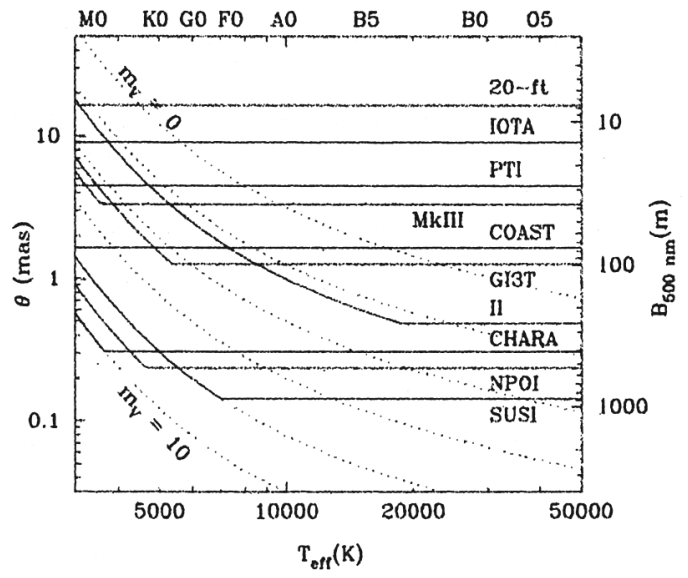

Figure 1. The regions of parameter space available to some of the current generation of optical interferometers. For each array, accessible stars are delimited by a horizontal boundary, below which the stars are unresolved at the maximum baseline, and a curve of constant magnitude, below which the star is to faint to be studied. The dotted curves show the apparent uniform disk size of stars of a given $v$ magnitude as a function of effective temperature. Observations at $\lambda=500 \mathrm{~nm}$ have been assumed.

infrared spectroscopic studies of Monnier et al. (2000), but in this review I will concentrate on the optical and near-infrared regime, since it is at these wavelengths that we can expect to see substantial instrumental advances being made in the very near future.

\section{Interferometric possibilities and limitations}

Bedding (this volume) has already presented a summary of the current generation of interferometers in operation and under construction. Before highlighting the results that have been obtained with these, it is worthwhile re-iterating the scope of these ground-based instruments for astronomy. The fundamental limitations of these existing arrays are summarised in Fig. 1, which shows the range of stellar types accessible to them. In each case, the science that is possible is constrained on the one hand by the maximum baseline, and hence limiting resolution, of the array and on the other by how faint a target can be successfully observed.

While arrays are already being commissioned with baselines as long as $450 \mathrm{~m}$, the principal shortcoming of this first generation of interferometers has been their poor sensitivity. As for adaptive optics systems, optical/IR interferometers have to detect a sufficient number of photons per atmospheric coherence time to allow tracking and continuous correction of the atmospheric phase fluctuations. The use of typically rather small $(<0.5 \mathrm{~m}$ diameter $)$ collectors in the first generation of instruments has thus been a significant hindrance to good sensitivity. At present, these limits are roughly at the $\sim 9$ mag level, though it is hoped that arrays such as the Keck and VLT interferometers will be able 
to track the atmospheric fringe motion using reference sources as faint as 12 th magnitude at $\mathrm{K}$.

A further limitation of many arrays has been the small number of interferometer elements. This has two main repercussions. First, it implies that measurements of the visibility function, i.e. of the Fourier transform of the source brightness distribution, will only be possible on a small number of baselines. Since each of these corresponds to a particular angular scale on the sky, the range of these that any interferometer will be sensitive to will in general be small unless the interferometer elements can be reconfigured or measurements can be made over a wide range of hour angles.

The second, and perhaps more important, consequence is the impact that a small number of collecting elements will have on the imaging capability of an array. As for radio VLBI arrays, interferometric imaging in the presence of atmospheric turbulence is only possible if simultaneous measurements of many visibility phases are secured. This allows closure phases, i.e. linear combinations of the visibility phases that remain unaffected by the atmosphere, to be constructed, and it is these, together with the self-calibration strategies developed in radioastronomy (Pearson \& Readhead 1984) that permit image recovery. The efficacy of these methods is a strong function of the total number of array elements, $N$, with the fraction of "missing" phase information not encoded in the closure phases being equal to $2 / N$. It is worth noting that only the COAST (Baldwin et al. 1994) and NPOI (Armstrong et al. 1998) arrays currently have any facility for measuring closure phases, though the CHARA, VLT and Keck interferometers will all have this capability at different levels eventually.

Table 2. An outline of the types of stellar science goals for optical/IR interferometers. This is by no means an exhaustive list and is based essentially on programmes that have already been initiated with the current generation of arrays.

\begin{tabular}{lr}
\hline General area & Detailed topics \\
\hline Fundamental parameters & Radii \& Effective temperatures \\
& Masses through binary orbits \\
Atmospheric studies & Limb-darkening \\
& Stratification of cool atmospheres \\
Dynamical studies & Stellar surface imaging \\
& Mira pulsations \\
Envelope studies & Cepheid pulsations \\
& Hot: e.g. Be star emission line nebulae \\
& Cool: dust shells round evolved systems \\
\hline \hline
\end{tabular}

The upshot of the preceding paragraphs is thus that we should expect existing interferometers to have made only modest advances in our understanding of stellar atmospheres. Nevertheless, it is certainly valuable to reflect on the range of possible topics that could, in principle, be explored. Table 2 lists some of these. At this juncture perhaps all that one need note is that the list is long, and that successes in these areas will undoubtedly have wider implications in our understanding of many other astrophysical phenomena, e.g. mass-loss, stellar convection, and the distance scale. 

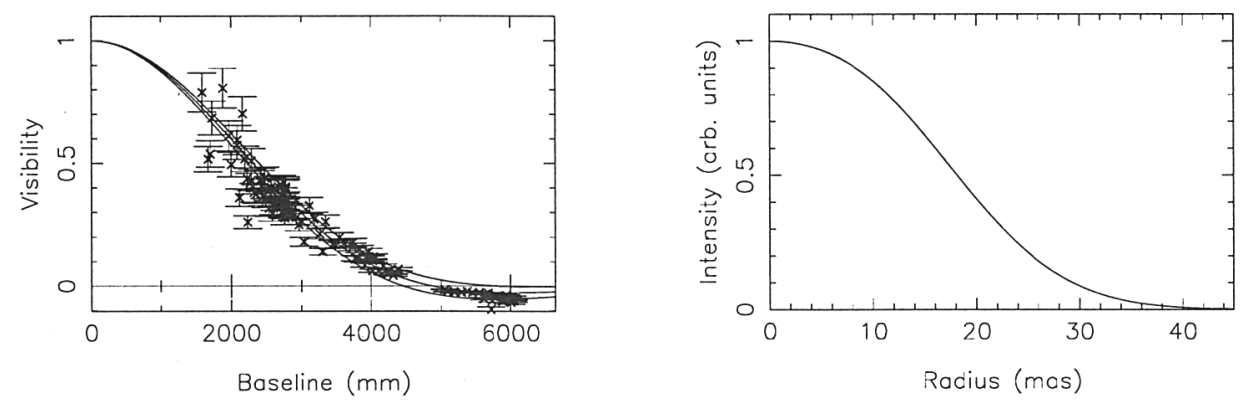

Figure 2. Visibility function (left) and inferred radial brightness distribution (right) for Betelgeuse, measured with the COAST interferometer at $850 \mathrm{~nm}$ (Burns et al. 1997).

\section{Results to date}

\subsection{Stellar diameter measurements}

Angular diameter measurements have long been a staple of high-angular resolution techniques. This remains true to date, with current campaigns having tended to concentrate on stars with diameters in the range 2-50 mas. These types of measurements are most conveniently performed in the near-infrared JHK bands, where the effects of limb-darkening are ostensibly weaker than in the optical. Usually spectral resolutions of order tens are used. Most importantly, these types of studies have now reached a very high level of precision through the use of single-mode fibre spatial filters (see e.g. Perrin et al. 1999) It is now not unusual for workers to quote formal errors of better than a percent for a diameter estimate, and it is the improvement in reliable calibration responsible for this that has been the most important development over the last 5 years.

Applications of these measurements have included better definition of the effective temperature scale of cool giants (Perrin et al. 1998; van Belle et al. 1999), the calibration of other diameter estimators (Alonso et al. 2000) and more generally the calibration of cool stellar structure codes.

\subsection{Limb-darkening studies}

The extension of uniform disk diameter measurements to investigations of the detailed radial brightness profiles of stars has seen somewhat less progress. In general, exploration of the detailed intensity profile requires observations in well defined spectral bandpasses well beyond the first null of the visibility function. The requirement of having well sampled visibility data extending to baselines where the source is well resolved, and therefore difficult to measure with good signal-to-noise, has meant that only a handful of stars have had their limbdarkening measured with interferometers in the past 5 years (Quirrenbach et al. 1996; Burns et al. 1997; Hajian et al. 1998).

Figure 2 shows a typical dataset, where in this instance the data have been used to distinguish between different limb-darkening approximations. In the future one can expect more detailed studies such as this being used to provide a direct check on stellar atmosphere codes, particularly for cooler systems, and im- 

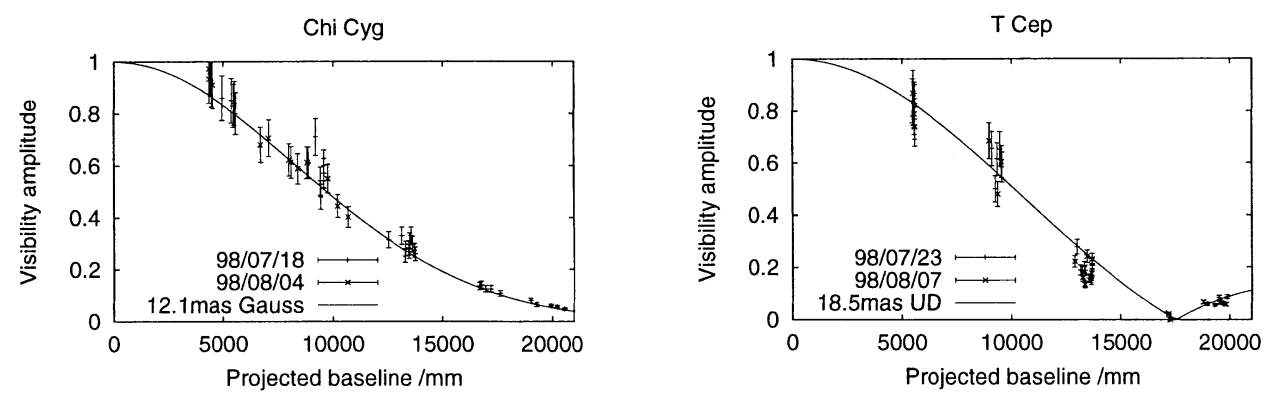

Figure 3. Observed visibility functions for two different Mira variables measured in the near-infrared with the COAST interferometer. The difference between the best-fit models - Gaussian for Chi Cyg and uniform-disk for T Cep - is striking (Young et al. in preparation).

proved limb-darkening parametrisations being used in the analysis of occultation and photometric studies of eclipsing binaries.

\subsection{Cool stellar atmospheres}

One of the most exciting synergies that has developed in recent years between interferometrists and stellar theoreticians has been in the area of cool stellar atmospheres. In particular, the detailed modelling of $M$ giant atmospheres, where the effects of limb-darkening and atmospheric extension are both important, has been championed by Scholz and his co-workers (see, e.g., Bessell, Scholz, \& Wood 1996; Hofmann \& Scholz 1998; Hofmann, Scholz, \& Wood 1998). These models have now developed to a stage where precision tests of the physics of the atmospheres can be made with suitable interferometric measurements.

More specifically, the need is for simultaneous multi-wavelength measurements to determine the apparent brightness profiles in and out of various molecular bands. Few studies have been able to combine the spectral $(R \sim 1000)$ and spatial resolutions required with good sensitivity, but first attempts at this have been presented by Jacob et al. (2000) and Hofmann et al. (2000). Hopefully, future studies like these will be able to explore the temperature and compositional stratification in these atmospheres, as well as the evidence for non-circular symmetry in the outer stellar envelopes of evolved stars.

\subsection{Stellar surface imaging}

As has been mentioned elsewhere, imaging with the current generation of stellar interferometers is a particularly challenging task. Not only is the necessary Fourier plane coverage difficult to realize - at least 5 collecting elements are typically needed to guarantee a moderate number of pixels across the stellar disk - but the rapid reduction in signal-to-noise as the source becomes resolved has meant that only the brightest supergiants have been targeted. Although the scientific payoffs are considerable, and include studies of convection, magnetic activity, mass loss, and the structure of the upper stellar atmosphere, the only star that has had its surface imaged with a separated-element optical interferometer to date is Betelgeuse (see, e.g. Young et al. 2000a). 
Interestingly, their multi-wavelength measurements suggest a different model for the ubiquitous hotspots seen on the surface of the star than had previously been proposed (see, e.g. Buscher et al. 1990; Wilson, Dhillon, \& Haniff 1997). Quite clearly there is a pressing need for future instruments to improve on the rudimentary imaging that has been achieved to date.

\subsection{Dynamical studies}

Improvements in reliability and availability have meant that routine monitoring of individual targets with long-baseline interferometers has now become feasible. One of the principal areas of interest here has been the direct measurement of stellar pulsation. This has been most effectively carried out on Mira and Cepheid variables.

For the former of these, the major aim has been to test the dynamical models that are expected to govern their mass loss. The most successful studies have been undertaken by the Cambridge group (see, e.g. Young et al. 2000b and references therein) who have detected long-term large amplitude modulations of the apparent stellar sizes of two Miras. The interpretation of these data, however, is complicated due to the possible contamination of the signals by emission from volatile molecular species whose concentrations are expected to vary with pulsation phase. As such, the first precise tests of existing dynamical models are still awaiting future campaigns that will use measurements in narrow "pure" continuum bands in near-infrared regions of the spectrum.

For Cepheids, the major goal has always been to measure their diameter changes directly so as to obtain an independent geometric calibration of the distance scale. Unfortunately, the small apparent diameters of even the brightest Cepheids ( $\sim$ a few mas) have meant that initial measurements on relatively short baselines have only been able to determine the mean diameters of these stars (Mourard et al. 1997; Nordgren et al. 2000). Very recently, however, Lane et al. (2000) have reported the first unambiguous measurement of Cepheid pulsation through interferometry using the Palomar Testbed Interferometer. With an estimated uncertainty of $15 \%$, their derived distance to $\zeta$ Geminorum is no better than earlier indirect measures, but they do expect significant improvements in the near future. If these are indeed realised, and the period-luminosity relation calibrated to better than a few per cent, there seems no doubt that optical/IR interferometry will have successfully entered the cosmological arena!

\subsection{Stellar envelopes}

The characterisation of resolved stellar envelopes is, in a similar manner to stellar surface imaging, one of the most challenging goals for optical/IR interferometry. Although space here permits only a cursory summary of the background to the area, it should be clear that the unambiguous determination of the geometry, asymmetry, and composition of both the gas and dust envelopes around even a small sample of active stars would be an outstanding achievement.

Most of the high-angular resolution work on the H- $\alpha$ emission nebulae of Be stars has originated from the GI2T group (see, e.g. Vakili et al. 1998; Stee et al. 1998) with additional important contributions by Quirrenbach et al. (1997). While these have been pivotal in better understanding these systems, unambiguous imaging in a variety of spectral lines remains the target for the near future. 
The most impressive recent advances in envelope imaging have come from using interferometric techniques with single large telescopes. Over the past two years Tuthill and co-workers (Tuthill, Monnier, \& Danchi 1999; Tuthill et al. 2000) have reported quite beautiful diffraction-limited images of dust nebulae obtained using non-redundant aperture-masking (Haniff et al. 1987; Buscher et al. 1990) at the Keck I telescope. Not only have these completely elucidated the geometry of the circumstellar dust in these sources, but multi-epoch maps have revealed the proper motions of this material. More interestingly, in each case, the observed geometric configuration of the dust bears little resemblance to the distribution inferred on the basis of earlier spectral energy distribution fitting. There can be no stronger argument for the need for high-angular resolution imaging than presented by these specific examples.

\section{Summary}

With time (and a page limit) upon us, conclusions must be brief. What is evident, I hope, is that spatially resolved studies on stellar and sub-stellar scales are already here, and that the future, with the prospect of a number of new facility interferometers being commissioned over the next few years, looks very promising. My two requests for the future, if I can be permitted to make them, are quite simple: (i) that observers demand facility interferometers with good spectroscopic and angular resolution, a rapid imaging capability, and good sensitivity, and that (ii) theoreticians do not let the observers start securing data that they are unable to interpret. The challenge falls on both communities, I hope both rise to it.

\section{References}

Alonso, A., Salaris, M., Arribas, S., Martinez-Roger, C., \& Asensio Ramos, A. 2000, A\&A, 355, 1060

Armstrong, J.T., Mozurkewich, D., Rickard, L.J., Hutter, D.J., Benson, J.A., Bowers, P.F., Elias, M.N., Hummel, C.A., Johnston, K.J., Buscher, D.F., Clark, J.H., Ha, L., Ling, L.-C., White, N.M., Simon, R.S. 1998, ApJ, 496,550

Baldwin, J.E., Boysen, R.C., Cox, G.C., Haniff, C.A., Rogers, J., Warner, P.J., Wilson, D.M., \& Mackay, C.D. 1994, Proc. SPIE, 2200, 118

Bedding, T.R. 2000, this volume

Bessell, M.S., Scholz, M., \& Wood, P.R. 1996, A\&A, 307, 481

Burns, D., Baldwin, J.E., Boysen, R.C., Haniff, C.A., Lawson, P.R., Mackay, C.D., Rogers, J., Scott, T.R., Warner, P.J., Wilson, D.M.A., \& Young, J.S. 1997, MNRAS, 290, 111

Buscher, D.F., Baldwin, J.E., Warner, P.J., \& Haniff, C.A. 1990, MNRAS, 245, $7 \mathrm{p}$

Haniff, C.A., Mackay, C.D., Titterington, D.J., Sivia, D., Baldwin, J.E. 1987, Nature, 328, 694

Hajian, A.R., Armstrong, J.T., Hummel, C.A., Benson, J.A., Mozurkewich, D., Pauls, T.A., Hutter, D.J., Elias, N.M., Johnston, K.J., Rickard, L.J., \& White, N.M. 1998, ApJ, 496, 484 
Hofmann, K.-H., \& Scholz, M. 1998, A\&A, 335, 637

Hofmann, K.-H., Scholz, M., \& Wood, P.R. 1998, A\&A, 339, 846

Hofmann, K.-H., Balega, Y., Scholz, M., \& Weigelt, G. 2000, A\&A, 353, 1016

Jacob, A.P., Bedding, T.R., Robertson, J.G., \& Scholz, M. 2000, MNRAS, 312, 733

Lane, B.F., Kuchner, M.J., Boden, A.F., Creech-Eakman, M., \& Kulkarni, S.R. 2000, Nature, 407, 485

Lim, J., Carilli, C.L., White, S.M., Beasley, A.J., \& Marson, R.G. 1998, Nature, 392,575

Monnier, J.D., Danchi, W.C., Hale, D.S., Tuthill, P.G., \& Townes, C.H. 2000, ApJ, 543, 868

Mourard, D., Bonneau, D., Koechlin, L., Labeyrie, A., Morand, F., Stee, P., Tallon-Bosc, I., \& Vakili, F. 1997, A\&A, 317, 789

Nordgren, T.E., Armstrong, J.T., Germain, M.E., Hindsley, R.B., Hajian, A., Sudol, J.J., Hummel, C.A. 2000, ApJ, 543, 972

Perrin, G., Coude du Foresto, V., Ridgway, S.T., Mariotti, J.-M., Traub, W.A., Carleton, N.P., \& Lacasse, M.G. 1998, A\&A, 331, 619

Perrin, G., Coude du Foresto, V., Ridgway, S.T., Mennesson, B., Ruilier, C., Mariotti, J.-M., Traub, W.A., \& Lacasse, M.G. 1999, A\&A, 345, 221

Pearson, T.J., \& Readhead, A.C.S. 1984, ARA\&A, 22, 97

Quirrenbach, A., Mozurkewich, D., Buscher, D.F., Hummel, C.A., \& Armstrong, J.T. 1996, A\&A, 312, 160

Quirrenbach, A., Bjorkman, K.S., Bjorkman, J.E., Hummel, C.A., Buscher, D.F., Armstrong, J.T., Mozurkewich, D., Elias, N.M.,\& Babler, B.L. 1997, ApJ, 479, 477

Stee, Ph., Vakili, F., Bonneau, D., \& Mourard, D. 1998, A\&A, 332, 268

Tuthill, P.G., Monnier, J.D., \& Danchi, W.C. 1999, Nature, 398, 487

Tuthill, P.G., Monnier, J.D., Danchi, W.C., \& Lopez, B. 2000, ApJ, 543, 284

Vakili, F., Mourard, D., Stee, Ph., Bonneau, D., Berio, P., Chesneau, O., Thureau, N., Morand, F., Labeyrie, A., \& Tallon-Bosc, I. 1998, A\&A, 335,261

van Belle, G.T., Lane, B.F., Thompson, R.R., Boden, A.F., Colavita, M.M., Dumont, P.J., Mobley, D.W., Palmer, D., Shao, M., Vasisht, G.X., Wallace, J.K., Creech-Eakman, M.J., Koresko, C.D., Kulkarni, S.R., Pan, X.P., \& Gubler, J. 1999, AJ, 117, 521

Wilson, R.W., Dhillon, V.S., \& Haniff, C.A. 1997, MNRAS, 291, 819

Young, J.S., Baldwin, J.E., Boysen, R.C., Haniff, C.A., Lawson, P.R., Mackay, C.D., Pearson, D., Rogers, J., St.-Jacques, D., Warner, P.J., Wilson, D.M.A., \& Wilson, R.W. 2000a, MNRAS, 315, 635

Young, J.S., Baldwin, J.E., Boysen, R.C., Haniff, C.A., Pearson, D., Rogers, J., St.-Jacques, D., Warner, P.J., \& Wilson, D.M.A. 2000b, MNRAS, 318, 381 